\title{
Dependence of Dirac eigenmodes on boundary conditions for SU(2) lattice gauge theory *
}

\author{
Christof Gattringer and Stefan Solbrig \\ Institut für Theoretische Physik, Universität Regensburg, 93040 Regensburg, Germany.
}

\begin{abstract}
We analyze zero modes of the Dirac operator for $\mathrm{SU}(2)$ lattice gauge theory. We find that the zero modes are strongly localized in all 4 directions. The position of these lumps depends on the boundary conditions we use for the Dirac operator. We compare periodic boundary conditions and anti-periodic boundary conditions and find that the position of the zero modes jumps for about one third of the configurations.
\end{abstract}

\section{Introduction}

In the last few years the study of Dirac eigenmodes was established as a powerful method for analyzing infrared structures of lattice gauge field configurations. After early work (e.g. [1]), the idea of probing the QCD vacuum with Dirac eigenmodes picked up steam with the discussion of the local chirality of near-zero modes $[2$. In 3 . evidence was given, that the infrared structures which couple to the low lying Dirac eigenmodes are self-dual, and in [4 it was argued, that these structures could be low-dimensional objects.

A new technique, the probing of the $\mathrm{QCD}$ vacuum with eigenmodes that are computed for different boundary conditions of the Dirac operator, was introduced for the study of so-called Kraan van Baal $(\mathrm{KvB})$ solutions [5]. KvB solutions are solutions of the classical Yang-Mills equations on a Euclidean cylinder. A KvB solution of topological charge $Q=1$ can be seen to consist of $N$ constituents (for $\mathrm{SU}(N)$ ), which locally resemble monopoles. For higher charges one finds $Q \times N$ constituents [6]. The connection to the Dirac eigenmodes was given in [7, where the zero mode $\phi$ for a charge $1 \mathrm{KvB}$ solution was calculated. This eigenvalue problem was solved for a general boundary condition in the compactified (time-) direction,

$\phi(x, \beta)=e^{i 2 \pi \zeta} \phi(x, 0)$,

where $\beta$ is the extent of the compact direction of

\footnotetext{
*Talk presented at QCD04 by C.G. This work was sup-
} ported by DFG and BMBF. the Euclidean cylinder. The boundary phase $\zeta$ has values between 0 and 1 (we stress that in all of our discussion the gauge fields obey periodic boundary conditions). It was shown, that the zero mode is not distributed over all of the constituents simultaneously, but instead is localized on only one of them. Which of the constituents is chosen depends on the phase $\zeta$, relative to the phases determining the Polyakov loop at spatial infinity. Thus if one varies $\zeta$, the zero mode will become located on each of the constituents, one after the other. This property of the zero mode was confirmed in lattice studies [8] and strong evidence for the appearance of $\mathrm{KvB}$ solutions in thermalized lattice gauge configurations was given. In [9] it was argued, that an alternative interpretation where the zero mode hops between integer charged objects is unlikely.

Subsequently the same type of analysis, i.e. using the boundary condition (1) to probe gauge configurations, was applied to $\mathrm{SU}(3)$ lattice gauge theory on the periodic torus [10]. Similar to the case of the Euclidean cylinder, it was observed, that the zero mode of a charge 1 configuration can be located at different positions, depending on the boundary condition. The observed zero modes are localized in all four directions. However, for the torus no analytic solutions are available, and the nature of the constituents is not known. The central question is whether also on the torus charge 1 configurations can have constituents. Attempts [1] to create localized charge 1 configurations with separated constituents on the torus with cooling have failed so far. How- 
ever, constituent-like structures in the cooled configurations are suggested by the behavior of the Polyakov loop observed in [11. Why these potential constituents are not separated in the cooled configurations is not clear.

In 12 zero modes for constant curvature configurations on the periodic torus were studied analytically. For SU(3) it was found that constant curvature configurations with charge 1 can be constructed such that the zero mode hops when the boundary condition is changed. Although this zero mode is not very strongly localized, constant curvature configurations cannot be a priori ruled out as candidates for explaining the findings of 10. For $\mathrm{SU}(2)$ the situation is different. It was shown that constant curvature $\mathrm{SU}(2)$ configurations only have even charge [12. Hence for $\mathrm{SU}(2)$, Ref. 12] rules out constant curvature configurations as candidates for explaining the hopping of the zero mode for charge 1 configurations. Thus it is interesting to know whether $\mathrm{SU}(2)$ configurations with charge 1 show zero modes that change their position as one changes the boundary condition. This question is addressed in this paper.

\section{Technicalities}

Our SU(2) gauge field configurations were generated with the Lüscher-Weisz action 13 on two different lattice sizes: $16^{4}$ with $\beta=1.95$ and $12^{4}$ with $\beta=1.90$. For both volumes we generated a large number of well decorrelated gauge field configurations. For the complete ensembles we calculated the 30 (50 for size $\left.12^{4}\right)$ smallest eigenvalues and the corresponding eigenvectors of the chirally improved lattice Dirac operator [14] with periodic boundary conditions. The chirally improved Dirac operator has good chiral properties and was shown [15] to optimally resolve the zero mode of a discretized instanton. We use the implicitly restarted Arnoldi method for calculating the eigenmodes.

From all configurations we subsequently selected the subset of configurations with a single zero mode. Thus, according to the index theorem, only configurations with topological charge $Q= \pm 1$ were chosen. Restricting to configurations with only a single zero mode, allowed us to avoid potential mixing of zero modes. Such a mixing could occur in configurations with higher charge, since a linear combination of different zero modes is again a zero mode. For such a mixture the hopping which we observe could be a hopping between two zero modes corresponding to integer charged objects. The restriction to configurations with a single zero mode excludes this simplest mixing mechanism, as opposed to mixing with near-zero modes.

For the selected subset we calculate the eigenmodes and eigenvectors a second time, now using boundary conditions that are anti-periodic in the 4-direction and periodic for the other three directions (we will refer to these mixed boundary conditions as "anti-periodic"). For most configurations in the subset we again find a single zero mode also for anti-periodic boundary conditions. There are, however, a few exceptions which we discuss for the $16^{4}$ ensemble: In this ensemble we have 105 configurations with a single zero mode for periodic boundary conditions. When switching to anti-periodic boundary conditions, for 4 of the 105 configurations we found three zero modes. In all the 4 cases one of the three modes had opposite chirality, such that the topological charge remained unchanged. Another configuration had four zero modes, such that the topological charge was different. These 5 configurations were omitted in the final analysis. This leaves us with 100 configurations on $16^{4}$ where we find exactly one zero mode for both boundary conditions. For $12^{4}$ we have 114 such configurations. These subsets were used for our analysis.

\section{Localization of the zero modes}

Let us begin the presentation of our results with an analysis of the scalar density of the zero modes. For a zero mode $\phi(x)_{\alpha, c}$, the scalar density $\rho(x)$ is defined as

$\rho(x)=\sum_{\alpha=1}^{4} \sum_{c=1}^{2}\left|\phi(x)_{\alpha, c}\right|^{2}$.

Here $\alpha$ and $c$ denote the Dirac and color indices. The scalar density is a gauge invariant object.

In order to demonstrate that the zero modes are localized in all four directions, in Figs. 1 and 

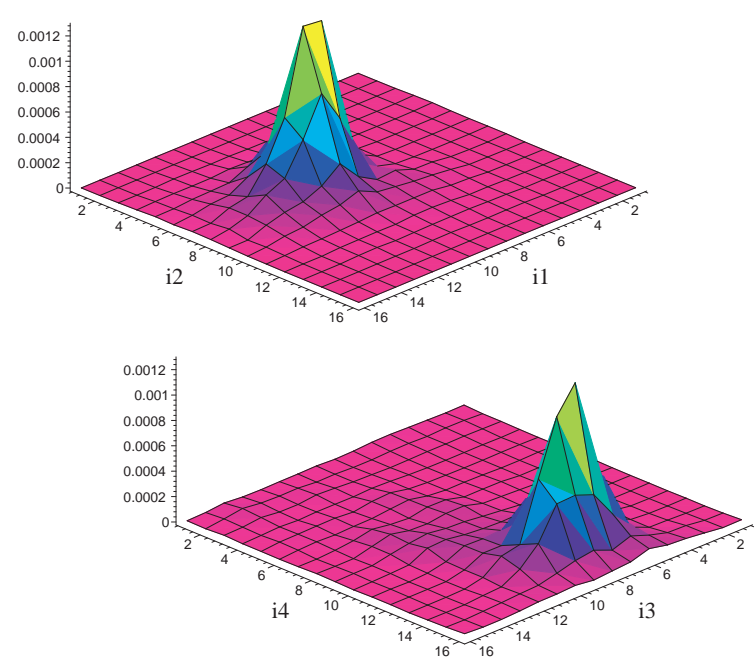

Figure 1. The pseudoscalar density for a zero mode with periodic boundary conditions. We show the 1-2 and 3-4 slices through the maximum of $\rho(x)$ which is located at $(10,7,7,13)$.

2 we show $\rho(x)$ for two different slices through the position of the maximum of the lump. In Fig. 1 this is done for the zero mode with periodic boundary conditions, in Fig. 2 for the antiperiodic boundary condition. Both figures are for the same configuration, but the positions of the zero modes with periodic and anti-periodic boundary conditions differ. For our example these positions are $x_{P}=(10,7,7,13), x_{A}=$ $(10,1,1,7)$. Thus the lumps seen by the periodic and the anti-periodic zero mode are 10.39 lattice spacings apart.

From the figures it is obvious, that both the periodic and the anti-periodic zero mode are localized in all directions. In particular we do not observe any special role of the 4-direction where we attached the anti-periodic boundary condition.

\section{Distribution of the lump separation}

In the last section we have discussed that some of the configurations have the periodic and the anti-periodic zero modes at different positions. In order to study this phenomenon in more detail, we analyze the distance $d$ between the maxima of

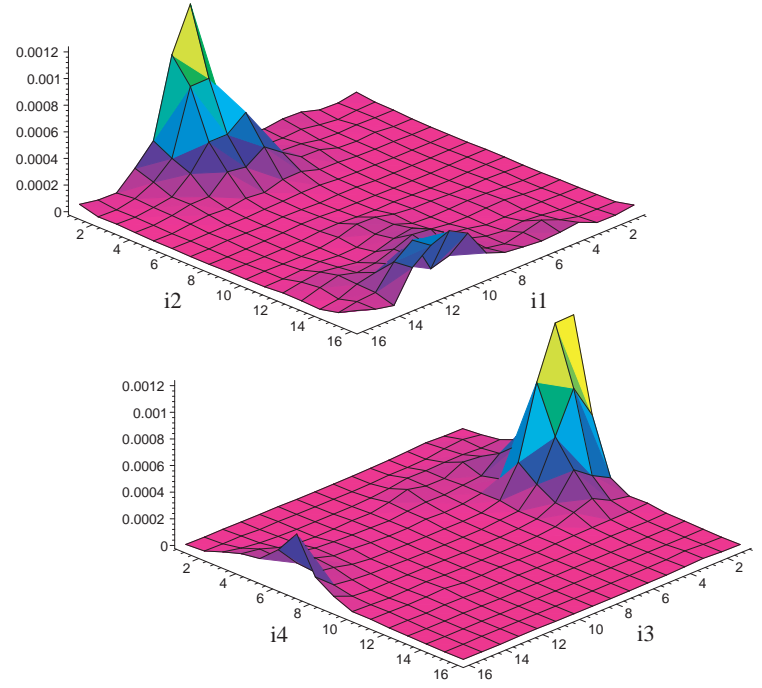

Figure 2. Same as Fig. 1, but now for the zero mode with anti-periodic boundary conditions. For these boundary conditions the maximum is located at $(10,1,1,7)$.

the two zero modes. We define

$d=\left\|x_{P}-x_{A}\right\|_{\text {torus }}$,

where $x_{P}, x_{A}$ are the positions of the maxima of $\rho(x)$ for the zero mode with periodic and anti-periodic boundary conditions. The distance $\|\ldots\|_{\text {torus }}$ is the minimal Euclidean distance on the torus, i.e. for each component of the vector we use the shorter of the two possible distances.

In Fig. 3 we show histograms for the distribution of $d$ for the two lattice sizes. We find that for both lattice sizes a large portion of the configurations have their periodic and anti-periodic zero modes at the same position, or only a small distance apart. However, both histograms show a second bump in the distribution at distances between $d=4$ and $d=12$. In particular we find that for the larger lattice 33 out of 100 configurations $(\sim 33 \%)$ have a $d$ larger than 3.5 lattice spacings. For the smaller lattice this is 27 out of 114 configurations $(\sim 24 \%)$. Thus for both lattice sizes we find that a sizable portion of the configurations show a different location of the zero modes for periodic and anti-periodic boundary conditions. This answers the question which we posed at the end of our introduction to the pos- 

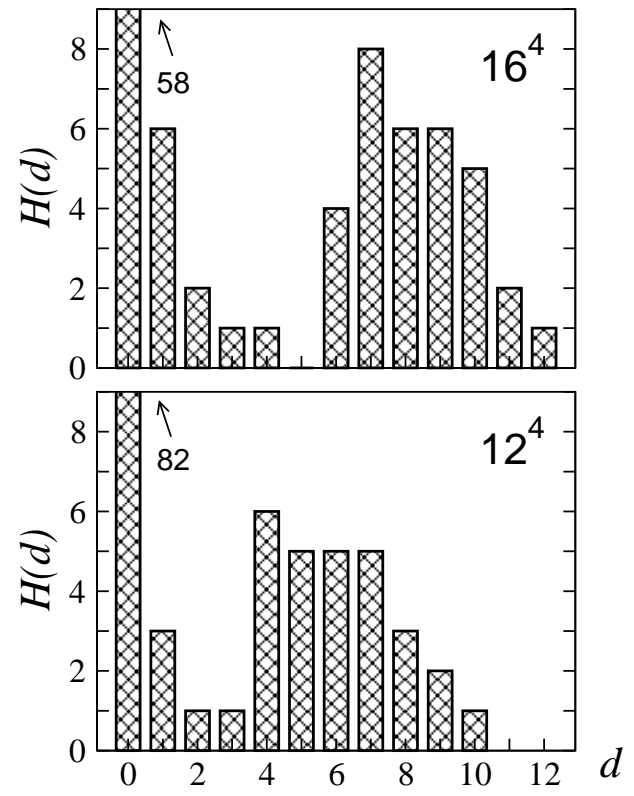

Figure 3. Histogram for the distribution of $d$.

itive, and we have established, that also $\mathrm{SU}(2)$ shows hopping zero modes when the boundary conditions are switched.

\section{REFERENCES}

1. T.L. Ivanenko and J.W. Negele, Nucl. Phys. Proc. Suppl. 63 (1998) 504; Ph. de Forcrand et al., Nucl. Phys. Proc. Suppl. 73 (1999); T. DeGrand and A. Hasenfratz, Phys. Rev. D 64 (2001) 034512; T. DeGrand, Phys. Rev. D 64 (2001) 094508.

2. I. Horváth et al., Phys. Rev. D 65 (2002) 014502, Phys. Rev. D 66 (2002) 034501; T. DeGrand and A. Hasenfratz, Phys. Rev. D 65 (2002) 014503; T. Blum et al., Phys. Rev. D 65 (2002) 014504; R.G. Edwards and U.M. Heller, Phys. Rev. D 65 (2002) 014505; I. Hip et al., Phys. Rev. D 65 (2002) 014506; C. Gattringer et al., Nucl. Phys. B 617 (2001) 101, (1985) Nucl. Phys. B 618 (2001) 205, Nucl. Phys. Proc. Suppl. 106 (2002) 492, P. Hasenfratz et al., Nucl. Phys. Proc. Suppl. 106 (2002) 751.

3. C. Gattringer, Phys. Rev. Lett. 88 (2002) 221601.

4. I. Horváth et al., Phys. Rev. D 68 (2003) 114505, Phys. Rev. D 67 (2003) 011501;
C. Aubin et al. [MILC], hep-lat/0410024.

5. T.C. Kraan and P. van Baal, Phys. Lett. B 428 (1998) 268, Phys. Lett. B 435 (1998) 389, Nucl. Phys. B 533 (1998) 627; K. Lee and C. Lu, Phys. Rev. D 58 (1998) 1025011.

6. F. Bruckmann and P. van Baal, Nucl. Phys. B 645 (2002) 105; F. Bruckmann, D. Nogradi and P. van Baal, Acta Phys. Polon. B 34 (2003) 5717 (hep-th/0309008); Nucl. Phys. B698 (2004) 233.

7. M. Garcia Pérez, A. González-Arroyo, C. Pena and P. van Baal, Phys. Rev. D 60 (1999) 031901; M.N. Chernodub, T.C. Kraan and P. van Baal, Nucl. Phys. Proc. Suppl. 83 (2000) 556; F. Bruckmann, D. Nogradi, P. van Baal, Nucl. Phys. B 666 (2003) 197.

8. E.-M. Ilgenfritz, B. V. Martemyanov, M. Müller-Preussker, S. Shcheredin and A. I. Veselov, Phys. Rev. D 66 (2002) 074503, Nucl. Phys. Proc. Suppl. 119 (2003) 754, C. Gattringer, Phys. Rev. D 67 (2003) 034507, C. Gattringer and S. Schaefer, Nucl. Phys. B 654 (2003) 30, C. Gattringer et al., Nucl. Phys. Proc. Suppl. 129 (2004) 653.

9. F. Bruckmann, M. Garcia Pérez, D. Nogradi and P. van Baal, Nucl. Phys. Proc. Suppl. 129 (2004) 727.

10. C. Gattringer and R. Pullirsch, Phys. Rev. D 69 (2004) 094510.

11. E. M. Ilgenfritz, B. V. Martemyanov, M. Müller-Preussker and A. I. Veselov, Phys. Rev. D 69 (2004) 114505; F. Bruckmann, E. M. Ilgenfritz, B. V. Martemyanov and P. van Baal, hep-lat/0408004

12. M. Garcia Pérez, Zero-Modes on constant curvature backgrounds, Talk presented at The QCD Vacuum from a Lattice Perspective, University of Regenburg, July 29-31, 2004.

13. M. Lüscher and P. Weisz, Commun. Math. Phys. 97 (1985) 59; G. Curci, P. Menotti and G. Paffuti, Phys. Lett. B 130 (1983) 205.

14. C. Gattringer, Phys. Rev. D 63 (2001) 114501; C. Gattringer, I. Hip and C. B. Lang, Nucl. Phys. B 597 (2001) 451.

15. C. Gattringer, M. Göckeler, C.B. Lang, P.E.L. Rakow and A. Schäfer, Phys. Lett. B 522 (2001) 194; C. Gattringer and C. B. Lang, Comput. Phys. Commun. 147 (2002) 398. 